\title{
An Approach for Rapid Prediction of Textile Draping Results for Variable Composite Component Geometries Using Deep Neural Networks
}

\author{
Clemens Zimmerling ${ }^{1,}$ a), Daniel Trippe ${ }^{1}$, Benedikt Fengler ${ }^{1}$, Luise Kärger ${ }^{1}$ \\ ${ }^{1}$ Karlsruhe Institute of Technology (KIT), Institute of Vehicle System Technology, Karlsruhe, Germany \\ a) Corresponding author: clemens.zimmerling @ kit.edu
}

\begin{abstract}
Continuous fibre reinforced plastics (CoFRPs) offer remarkable mechanical properties at low density and have thus drawn increasing attention in weight-sensitive industries over the last decades. Contrasting metals, manufacturing of CoFRPs consists of multiple steps, often comprising a forming process of a textile (draping). However, managing the inherently complex, anisotropic and non-linear material behaviour during textile forming and avoiding forming defects is a great challenge in serial production. To assess formability prior to manufacture, virtual process simulations can be applied. For optimum part quality, component design and applied process parameters must complement each other, which in turn requires a high number of optimisation iterations and quickly exceeds reasonable computation times. Considerable effort has been made with respect to obtaining optimum process parameters, however considering geometry adaptions to achieve manufacturability is rarely addressed. Deep Learning techniques using convolutional neural networks (CNN) are capable of learning complex system dynamics from supplied samples. In the work presented here, CNNs are used to rapidly predict textile forming results of variable component geometries. A large database of highly variant geometries and corresponding draping examples is generated, on which the CNNs are trained. The paper shows, that CNNs are capable of reproducing the underlying forming dynamics and that they generalise well to unknown test geometries. Contrasting traditional metamodel approaches, the presented method estimates not just a scalar part quality attribute, but predicts the complete shear strain field, which facilitates engineering interpretation. The method is demonstrated on different geometries ranging from simple shapes to complex geometries. Being computational inexpensive, CNNs give immediate feedback for real-time geometry iterations during component design. Thus, $\mathrm{CNNs}$ are considered a promising and time-efficient tool to reflect manufacturability during part and process design.
\end{abstract}

\section{INTRODUCTION}

Owing to their superior weight-specific mechanical properties, continuous fibre reinforced plastics (CoFRP) continue to attract attention in lightweight applications. Overall, the structural performance of a CoFRP component is a complex interaction of material properties and component geometry. For both, optimisation approaches have been studied in the past. For example, [1] and [2] address ply stack optimisation and patch optimisation for a fix geometry, while [3] focuses on local geometry adjustment for improved structural performance. Typically, current structural optimisation approaches assume perfect manufacturability and do not reflect potential defects. However, the presence of manufacturing defects can significantly impair the load bearing capacity, as exemplarily studied in [4]. Thus, manufacturability needs to be addressed during part and process design.

Manufacturing CoFRP components comprises multiple steps and often involves a forming process ("draping") of an initially flat textile, e.g. woven fabric or unidirectional non-crimp fabric. Upon tool closure, the fabric conforms to the shape of the tools. Compared to their high tensile stiffness in fibre direction, fabrics show a low shear resistance, which makes in-plane shear the predominant deformation mechanism during forming. As any other material, fabrics cannot deform infinitely but show material dependent forming limits. Since excessive shear increases the likelihood of unwanted defects, e.g. wrinkling or poor resin infiltration, the forming process requires particular attention. A frequently encountered measure to quantify the draping quality is the maximum shear angle in the fabric $\Omega$ after forming $\gamma_{12}^{\max }=\max _{\Omega}\left|\gamma_{12}\right|$, while lower shear angles are generally preferable. 
Physically motivated simulation techniques by Finite Elements (FE) are applied to predict and possibly optimise the forming process [5]. More recently, transferring information from forming simulations (e.g. local fibre orientations) to subsequent infiltration and curing simulations in a virtual process chain is increasingly proposed [6] for enhanced accuracy. To accurately capture the anisotropic and strongly non-linear nature of the material, a profuse amount of numerical expertise for model set up and considerable computational resources during simulation run are required. Although promising high physical accuracy, this makes iterative process optimisation approaches, see e.g. [7], and numerous component design variations time-consuming and in many cases impracticable. As a result, timeefficiency and ease of use are key aspects in manufacturability analysis, especially at early stages of the design process.

Different approaches to time-efficiently assess formability have emerged, such as general design guidelines [8], analytical relations for simple shapes [9] or kinematic draping approaches [10]. Yet, these approaches typically disregard material properties and process conditions (friction, blank holder forces) which delimits their significance. With the advent of elaborate Machine Learning (ML) techniques, an additional approach to rapid manufacturability assessment becomes conceivable. Instead of formulating and solving physics-based constitutive equations, ML in textile forming aims at identifying inherent patterns between input (geometry) and corresponding output (shear angles) within a data base of example draping simulations.

Previous studies show, that ML-models are able to model the forming behaviour for variable process conditions (blank holder forces) and geometry variations falling into an pre-specified scheme [11],[12]. However, these approaches are component-specific or focus on pre-specified geometry features only. Thus, they require generalisation to general component shapes, which is aspired in this work: An ML-model 'learns' the forming dynamics from an extensive set of highly-variable geometries. After training, the ML-model is requested to estimate the forming result of 'unknown' components, that are not part of the training-database. Specifically, a flat plate, a cap-profile and the double-dome geometry are studied. The predictions are validated against actual draping simulations and found to be in agreement. Hence, ML-techniques are deemed a promising tool for time-efficient part and process design.

\section{MODELLING APPROACH}

\section{Conceptual View}

Formally, this work treats a forming simulation as a function $\varphi_{\text {sim }}: G \mapsto \Gamma$, which maps from an input geometry $\underline{g} \in G$ to an output forming result $\underline{\gamma}_{12} \in \Gamma$ (shear angles). In general, the evaluation of $\varphi_{\text {sim }}$ is expensive, wherefore a numerical efficient substitute function $\mu_{\mathrm{ML}}: G \mapsto \Gamma$ is sought to approximate $\varphi_{\text {sim }}$. In general, $\varphi_{\text {sim }}$ can be evaluated for a given geometry $\underline{g} \in G$ (i.e. simulation run), but an explicit formulation of $\varphi_{\text {sim }}$ is not tractable ('black box'). Under these conditions, analytical approximation techniques, such as series expansion, are not applicable and only data-driven approximations are eligible. The model function $\mu_{\mathrm{ML}}$ stems from a function class $\mathcal{M}$ and is trained on a database $\boldsymbol{D}^{n}=\left\{\left(\underline{g}, \underline{\gamma_{12}}\right)_{1}, \ldots\left(\underline{g}, \underline{\gamma_{12}}\right)_{n}\right\}$ with $n$ draping examples. Different function classes are available, while polynomials, splines, Gaussian Regression, support vector machines or artificial neural networks (ANN) are frequently employed.

Owing to their extraordinary modelling capacity, ANNs show characteristics of universal approximators. That is, given sufficient training data, they can represent any function regardless of its complexity. They are organised in a hierarchical layer-structure with each layer carrying a number of neurons. In feedforward networks as used in this work, neurons of neighbouring layers are interconnected and transfer data through weighting, biasing and summation operations, which activate or deactivate neurons of subsequent layers. Thereby complex activation patterns form within the network. Ultimately, the last layer converts the patterns to an output quantity for engineering interpretation.

During model training, weights and biases of the neurons' interconnections (i.e. model parameters $\underline{\theta}$ ) are adjusted to match the supplied database. That is, $\underline{\theta}$ is tuned to minimise the loss function $L\left(\hat{\gamma}_{12}, \underline{\gamma}_{12}\right)$ between predicted output $\underline{\hat{\gamma}}_{12}(\underline{\theta}, \underline{g})$ and 'true' output $\underline{\gamma}_{12}(\underline{g})$ for a given input $\underline{g}$. Formally, this is cast as

$$
\underline{\theta}^{*}=\arg \min _{\underline{\theta} \in \mathbb{R}} L\left(\underline{\hat{\gamma}}_{12}, \underline{\gamma}_{12}\right) \text {. }
$$

While different loss functions $L\left(\hat{\gamma}_{12}, \underline{\gamma}_{12}\right)$ are applicable in general, this work uses the $\mathrm{L}_{2}$-loss defined by

$$
L=L_{2}=\frac{1}{n} \sum_{i=1}^{n} L_{2 i}=\frac{1}{n} \sum_{i=1}^{n}\left(\underline{\hat{\gamma}}_{12} i \underline{\gamma}_{12}\right)^{2}
$$


also known as mean squared error (MSE). It is common practice to split $\boldsymbol{D}^{n}$ into two non-intersecting subsets, where $\boldsymbol{V}^{n_{V}} \cup \boldsymbol{T}^{n_{T}}=\boldsymbol{D}^{n}$ and $\boldsymbol{V}^{n_{V}} \cap \boldsymbol{T}^{n_{T}}=\{\}$ with $n_{T}+n_{V}=n$. Parameter optimisation is performed on the training set $\boldsymbol{T}^{n_{T}}$ only, while the loss on the 'unknown' validation subset $\boldsymbol{V}^{n_{V}}$ gives an assessment of the generalisation capabilities of the ANN. In this work, $10 \%$ of $\boldsymbol{D}^{n}$ are held out as a validation split, i.e. $0.1=n_{V} / n$.

\section{Workflow and Data Sampling}

\section{Overview}

Figure 1 shows a sketch of the employed workflow. Initially, draping simulations on a set of geometry variants from a parametric CAD-model are performed. The geometries $\underline{g}^{i=1 \ldots n}$ and the obtained draping results $\underline{\gamma_{12}}{ }^{i=1 \ldots n}$ are collected in the database $\boldsymbol{D}^{n}$. The network architecture (number of neurons and layers etc.) is a-priori specified by the user. Subsequently, model training is performed on the training data $\boldsymbol{T}^{n_{T}} \subset \boldsymbol{D}^{n}$ by optimisation of the model parameters $\underline{\theta}$. Since loss-function gradients can be effectively computed using the backpropagation method, ANNtraining employs first-order gradient-based techniques, such as stochastic gradient descent and its various extensions. Once the model is trained, a new geometry $\tilde{g}$ can be evaluated for the forming result.

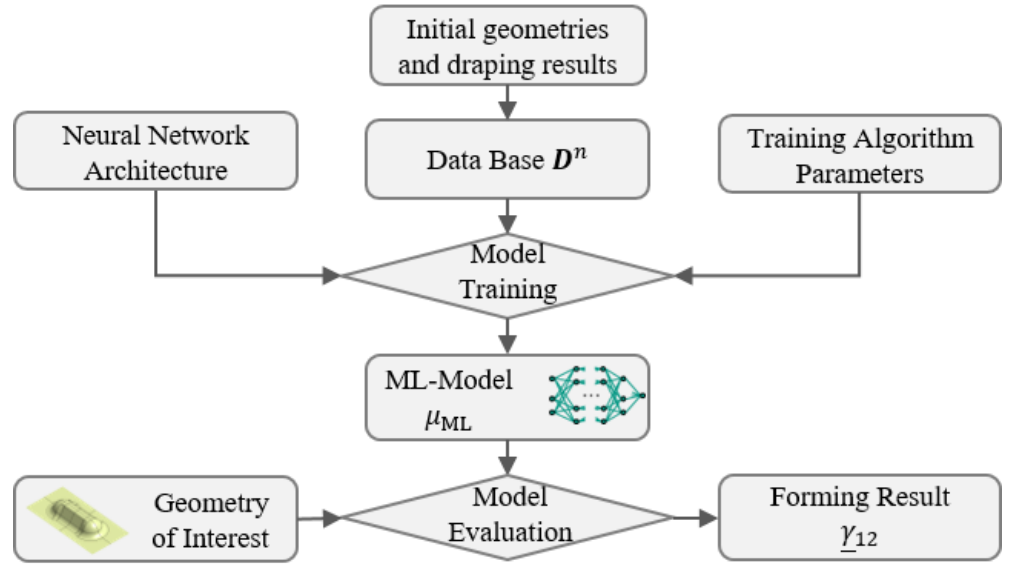

FIGURE 1: General workflow to obtain an ML-model for forming result estimation as used in this work.

\section{Data Acquisition}

In this work, the above workflow is applied to make full-field predictions of the shear angle distribution of selected convex test geometries. To this, a database $\boldsymbol{D}^{n}$ with a sufficient number of training examples is required. For maximum informative value on the forming dynamics, $\boldsymbol{D}^{n}$ needs a high variance of geometries. In this study, an elliptic CADgeometry is parameterised for automated geometry generation as presented in Table 1.

TABLE 1: Geometry parameterisation scheme and range for training data generation.

\begin{tabular}{|c|c|c|c|c|}
\hline Parameterisation scheme & Symbol & Meaning & Range & Example geometries \\
\hline & $l_{\min }$ & Length minor axis & $10 \ldots 200 \mathrm{~mm}$ & \\
\hline & $l_{\text {maj }}$ & Length major axis & $10 \ldots 200 \mathrm{~mm}$ & \\
\hline & $r_{\text {top }}$ & Radius (top) & $5 \ldots 90 \mathrm{~mm}$ & \\
\hline & $r_{\text {bot }}$ & Radius (bottom) & $5 \ldots 90 \mathrm{~mm}$ & \\
\hline & $\alpha$ & Draft Angle & $10 \ldots 70^{\circ}$ & \\
\hline & $h$ & Height & $10 \ldots 100 \mathrm{~mm}$ & \\
\hline
\end{tabular}

Through parameter variation a wide scope of different shapes is accessible, which ranges from strongly stretched elliptic profiles to cylinders. Thereby geometries with areas of nearly single-curvature (stretched ellipse) and numerous combinations of double-curvature are generated. To ensure uniform sampling of the parameter space, Latin 
Hypercube Sampling (LHS) is used, which is, amongst others, an established Design of Experiments strategy for parameter space exploration in numerical studies. To ensure a sufficiently large database in the first place, a total number of $n=10000$ LHS-samples are drawn and the according geometries generated.

For each geometry, draping simulations are performed. Although physics-based FE-simulations are more accurate, the authors choose a kinematic draping approach for two reasons: Most important, this reduces the numerical effort for training data generation to a reasonable amount for this preliminary feasibility analysis. Additionally, it beneficially precludes potentially misleading overlay effects from e.g. material non-linearities and allows thus for an isolated study of the network's predictive capability vis-à-vis variable geometries.

\section{Data Representation}

To avoid collision during tool-closure in punch-die-processes, all formable geometries must necessarily be undercut-free. Therefore, a bijective mapping of the geometries into the tool plane is tractable. Similar to a topographic map, the elevation $z(x, y)$ above the tool plane fully represents the geometry without loss of rigor. This allows an image-based geometry representation as exemplarily shown in Figure 2 (left), in which the local height is encoded by grayscale-values (GS). GS range from 0 to 255, whereby 0 GS ('black') denotes $h=0 \mathrm{~mm}$ and 255 GS ('white') corresponds to $h=h_{\max }=100 \mathrm{~mm}$. Apart from a convenient dimensional reduction from 3D to 2D, this representation grants access to a number of ML-techniques specialised in Computer Vision, e.g. object recognition, classification, tracking and modification.

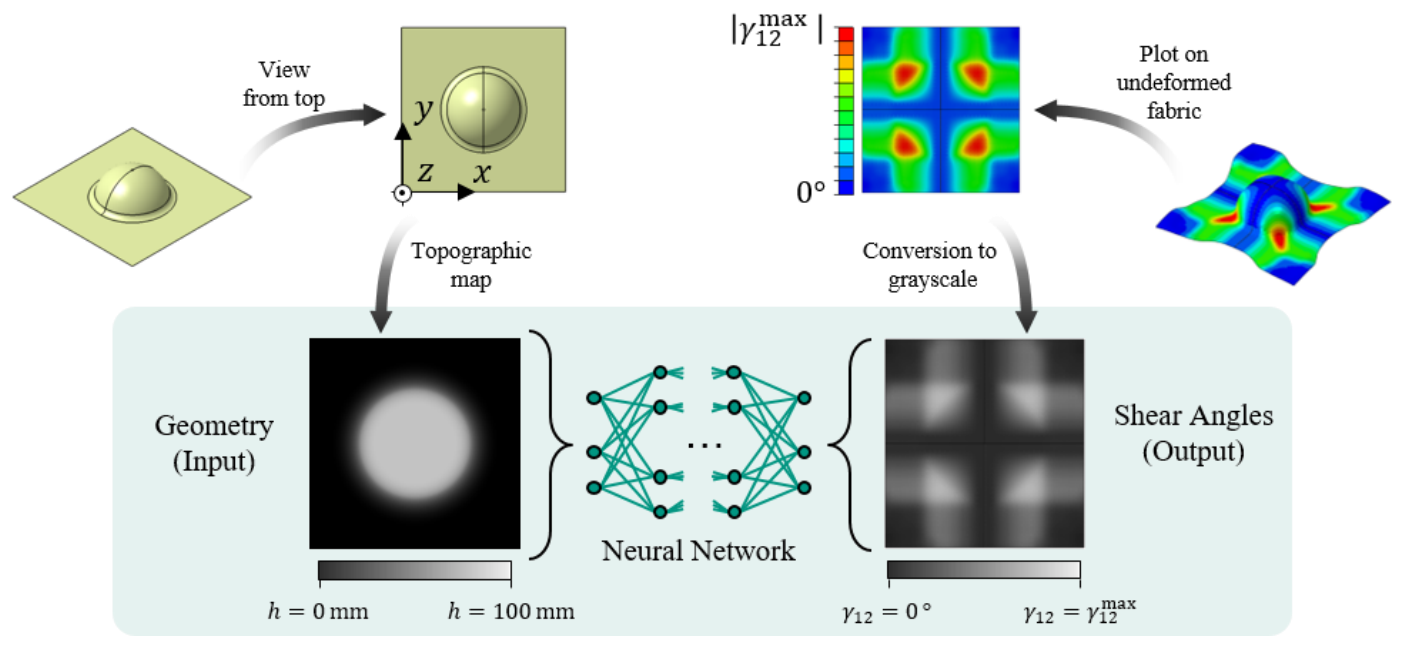

FIGURE 2: Data Representation for Neural Network Training and Evaluation.

Analogously, an image-based representation of the forming result can be established (Figure 2 right). Since the fabric is discretised into elements for the draping simulation, each fabric element $e$ is assigned a shear angle $\gamma_{12}^{e}$ after forming. The entirety of shear angles $\underline{\gamma}_{12}=\left(\gamma_{12}^{1}, \gamma_{12}^{2}, \ldots\right)$ in the fabric domain $\Omega$ constitutes the forming result. Plotting them onto the undeformed (i.e. flat) fabric yields a $2 \mathrm{D}$ representation of the forming result. Both, the geometry-image (input) and the forming result (output) are parsed to a $\mathrm{CNN}$ for end-to-end learning of the forming dynamics.

\section{Neural Network Training and Validation of Prediction Quality}

In fabric forming, a close spatial relation between geometry-curvature and the formation of shear angles is observed. Convolutional Neural Networks (CNNs), a type of feedforward networks, are specifically designed to take advantage of such spatially structured data [13] and are thus used in this work. As the name hints, CNNs slide ('convolve') a pre-specified number of templates ('kernels') across an input image. If template and image match at specific locations, according neurons in the next layer are activated and a so-called 'feature map' forms. The convolution process is repeated for the next network-layers, which form higher-order feature maps. In essence, these higher-order features are a compressed ('encoded') representation of draping-relevant information. Additional layers are subsequently added to decode and convert the compressed information to forming results by repeated transposed convolution operations, also known as "deconvolution". The network structure is schematically sketched in Figure 3. 


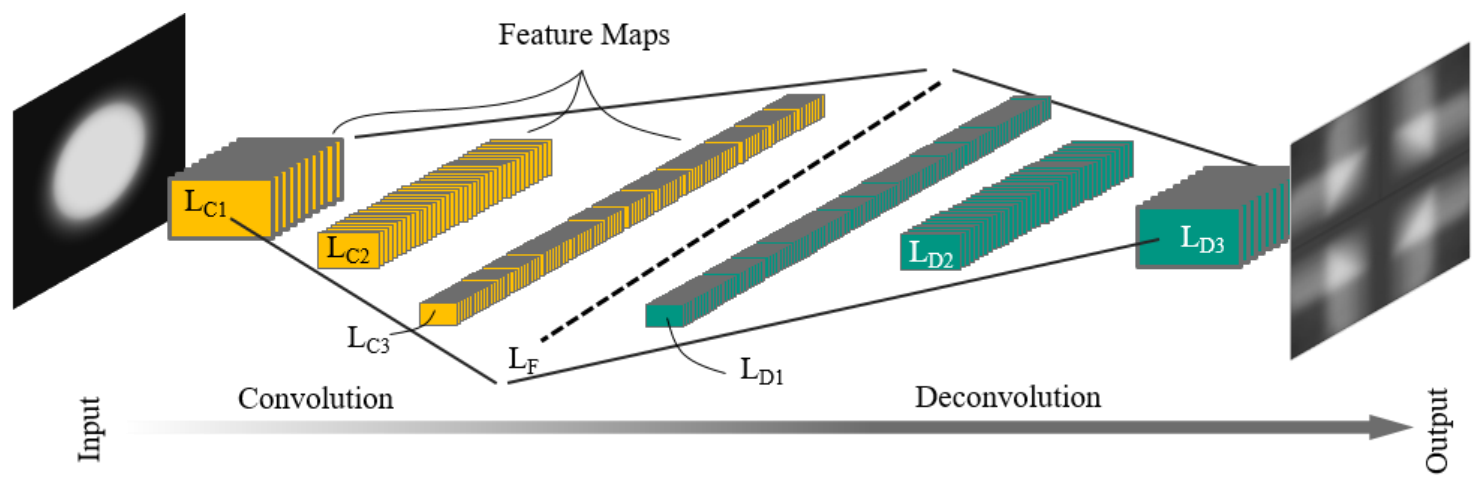

FIGURE 3: Architecture of the Neural Network with a Convolution-Deconvolution-structure. The layers $\mathrm{L}_{\mathrm{C} 1 \ldots 3}$ perform convolutions, $\mathrm{L}_{\mathrm{D} 1 \ldots 3}$ do deconvolution operations. A fully connected layer $\mathrm{LF}_{\mathrm{F}}$ connects convolutional and deconvolutional layers.

The sketched network is trained on the generated database $\boldsymbol{T}^{n_{T}}$ with $n_{T}=9000$ samples using the Adam-training algorithm, a variant of stochastic gradient descent. After network training, a remaining root mean square error RMSE $=$ $\sqrt{L} \approx 2.2^{\circ}$ is found on the held out validation set $\boldsymbol{V}^{n_{V}}$, which is deemed acceptable for application to test geometries.

\section{RESULTS ON TEST GEOMETRIES}

Of utmost interest is the network's capability to make predictions for geometries that lie outside the parameterisation scheme delineated above, i.e. non-elliptical geometries. Grouped by their curvature, three different shapes are investigated, a plate (zero curvature), a cap profile (single-curvature) and the double-dome (simple and double curvature). The results are presented in Table 2 with the performance metrics Root Mean Squared Error (RMSE) and the Mean Absolute Error (MAE) between CNN prediction and kinematic draping (KD) simulation. The variable $\Delta \gamma_{12}^{\max }$ denotes the difference of the maximum shear angles, i.e. $\Delta \gamma_{12}^{\max }=\gamma_{12_{\mathrm{ANN}}}^{\max }-\gamma_{12_{\mathrm{KD}}}^{\max }$, and $\%_{\text {corr }}$ quantifies the proportion of 'correctly' estimated pixels that deviate by max. $3^{\circ}$ from the kinematic draping simulation.

TABLE 2: Visualisation of the test geometries, their topographic maps and the shear strain distribution predicted by the CNN and a draping simulation on 'unknown' test geometries. Performance metrics for quantitative assessment are given on the right.

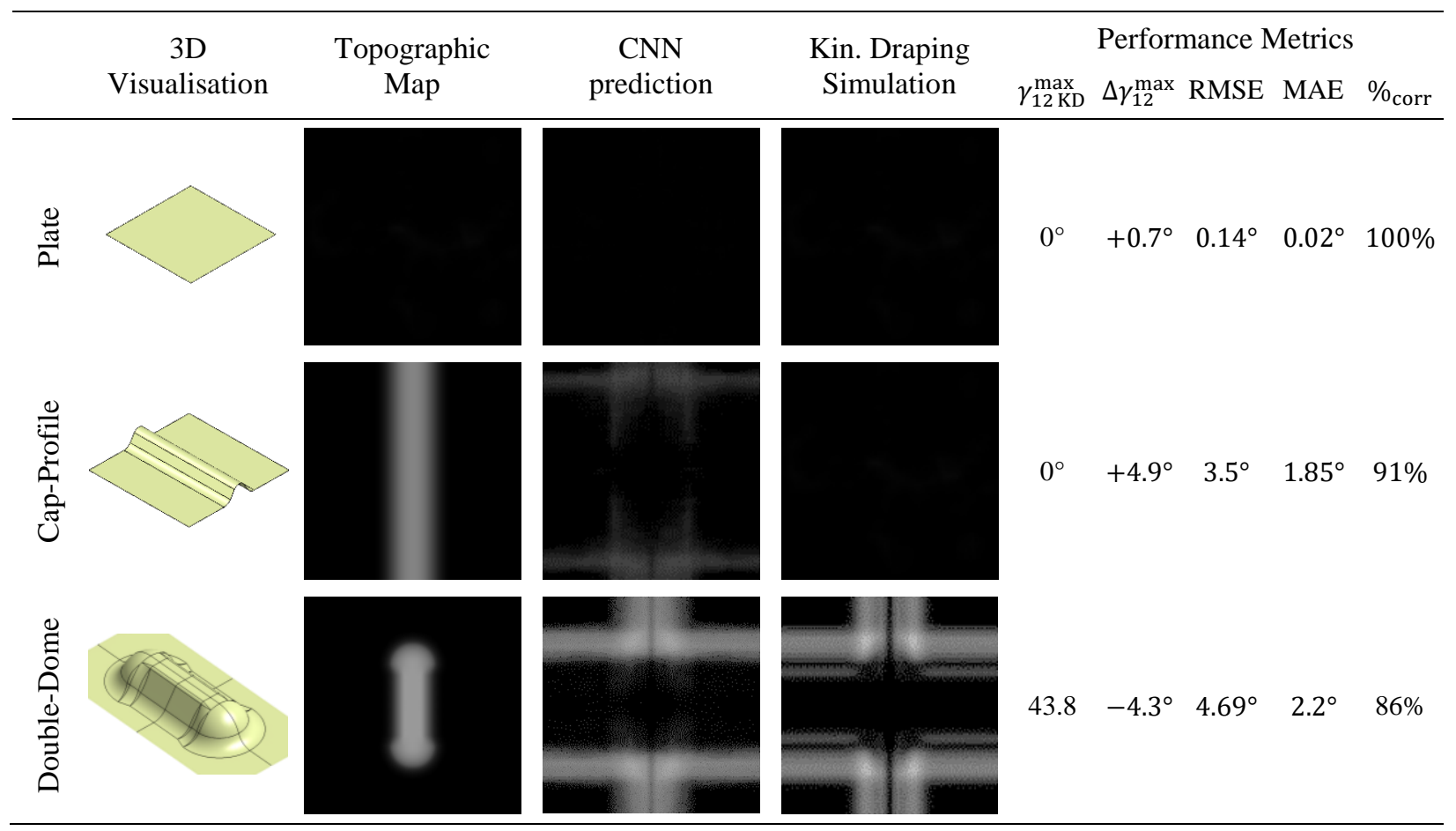


Since the plate and the cap profile pose no double-curvature, no shear deformations occur in the draping simulation $\left(\gamma_{12 \mathrm{KD}}^{\max }=0^{\circ}\right.$, fully black image). Compared to the CNN-estimation, an excellent agreement is found for the plate. The network's prediction is zero almost everywhere and deviates by $0.7^{\circ}$ at maximum, which is interpreted as numerical noise. For the cap profile, a good general agreement is observed, however, at the top and bottom minor deviations become apparent as barely visible bright areas. In these regions, a maximum deviation of $4.9^{\circ}$ is found. For the doubledome geometry, the shear strain distribution is well captured in general with $\Delta \gamma_{12}^{\max }=4.3^{\circ}$. However, a larger RMSE and MAE is observed. Upon closer inspection, additional bright lines of shear deformation occur in the kinematic draping simulation, which do not appear in the CNN-prediction. These narrow bands form due to the local concave double-curvature of the double-dome. However, as solely convex ellipses are included in the training data but no concave regions, the network cannot identify concave regions as relevant for shear strains. Thus, these missing shear strains increase RMSE and MAE although $\gamma_{12}^{\max }$ is captured acceptably well.

\section{CONCLUSION}

The proposed approach investigates a formability assessment of CoFRP components by evaluation of pre-trained ML-models as a time-efficient alternative to numerically expensive simulations. As shown in this work, convolutional neural networks $(\mathrm{CNN})$ are capable of learning the system dynamics of textile forming processes. Contrasting conventional meta-modelling strategies, the proposed approach gives full-field predictions of the forming result instead of lower-dimensional draping quality metrics. Furthermore, this work shows that CNNs are able to generalise the learnt forming behaviour to geometries that lie outside the initial training data. However, at the same time the importance of an appropriate training database becomes apparent: CNNs can only make valid predictions within the range of previously seen geometry classes (e.g. convex shapes). Therefore, future training databases require the deliberate inclusion of additional geometry shapes for full generalisation, e.g. convex-concave shapes.

Conceptually, ML-models are also applicable to process simulations other than forming, such as mould filling or resin curing. In conjunction with training data from physics-based simulations, such ML-approaches could ultimately lead to an easy-to-use tool for engineers that provides real-time feedback on manufacturability during design iterations. Although the effort to build an extensive training database from physics-based simulations is considerable, the benefits of immediate feedback for potentially arbitrary geometries may outweigh the expenses in the long term. Overall, ML-techniques appear promising for fast and close-to-reality component evaluation at early stages of the design phase.

\section{ACKNOWLEDGMENT}

The authors would like to thank the German Federal State Ministry of Science, Research and the Arts of Baden-Württemberg (MWK) for funding the project "Forschungsbrücke Karlsruhe-Stuttgart", which the presented work is carried out for. Additionally, the authors would like to thank the project partners at the Institute of Aircraft Design (IFB) of Stuttgart University, lead by Prof. Peter Middendorf for fruitful collaboration and discussion. The work is also part of the Young Investigator Group (YIG) "Tailored Composite Materials for Lightweight Vehicles", generously funded by the Vector Stiftung.

\section{REFERENCES}

[1] G. Allaire, G. Delgado, Journal of the Mechanics and Physics of Solids, 97, pp. 168-196 (2016)

[2] B. Fengler, L. Kärger, F. Henning, A. Hrymak, Journal of Composite Science, 2, 2, (2018)

[3] J. Paz, J. Díaz, L. Romera, M. Costas, Composite Structures, 133, pp. 499-507 (2015)

[4] L. Kärger, S. Galkin et al., Composite Structures, 192, pp. 143-152 (2018)

[5] P. Bussetta, N. Correia, Composites Part A, 113, pp. 12-31 (2018)

[6] L. Kärger, A. Bernath et al., Composite Structures, 132, pp. 350-358, (2015)

[7] S. Chen, O.P.L. McGregor et al., Composite Structures, 201, pp. 570-581 (2018)

[8] V. Butenko, A. Albers, Procedia CIRP, 70, pp. 41-46 (2018)

[9] R. Robertson, T.-J. Chu et al, Composites Part A, 31, pp. 703-715 (2000)

[10] A. K. Pickett, G. Creech, P. de Luca, Revue Européenne des Éléments Finis, 14, pp. 677-691 (2005)

[11] J. Pfrommer, C. Zimmerling et al., J. Beyerer, Procedia CIRP, 72, pp.426-431 (2018)

[12] C.Zimmerling, D. Dörr, F. Henning, L. Kärger, AIP Conference Proceedings, 1960, 020042 (2018)

[13] I. Goodfellow, Y. Bengio, A. Courville, Deep Learning (MIT Press,Cambridge,USA,2016). pp. 326-366. 